\title{
Determinants of survival in advanced non-small cell lung cancer patients treated with anti-PD-1/PD-L1 therapy
}

\author{
Wael Abdullah Sultan Ali ${ }^{1 \#}$, Pan Hui ${ }^{1 \#}$, Yuxiang $\mathrm{Ma}^{1 \#}$, Yuehan $\mathrm{Wu}^{2}$, Yang Zhang ${ }^{2}$, Yukun Chen ${ }^{1}$, \\ Shaodong Hong ${ }^{1}$, Yunpeng Yang ${ }^{1}$, Yan Huang ${ }^{1}$, Yuanyuan Zhao ${ }^{1}$, Wenfeng Fang ${ }^{1}$, Hongyun Zhao ${ }^{2}$, \\ Li Zhang ${ }^{1}$
}

${ }^{1}$ Department of Medical Oncology, Sun Yat-sen University Cancer Center, State Key Laboratory of Oncology in South China, Collaborative Innovation Center for Cancer Medicine, Guangzhou, China; ${ }^{2}$ Department of Clinical Research, Sun Yat-sen University Cancer Center, State Key Laboratory of Oncology in South China, Collaborative Innovation Center for Cancer Medicine, Guangzhou, China

Contributions: (I) Conception and design: WAS Ali, Y Ma, H Zhao, L Zhang; (II) Administrative support: H Zhao, L Zhang; (III) Provision of study materials or patients: Y Ma, H Zhao, L Zhang; (IV) Collection and assembly of data: WAS Ali, P Hui, Y Huang, Y Zhang; (V) Data analysis and interpretation: WAS Ali, Y Ma, P Hui; (VI) Manuscript writing: All authors; (VII) Final approval of manuscript: All authors.

"These authors contributed equally to this work.

Correspondence to: Li Zhang. Department of Medical Oncology, Sun Yat-sen University Cancer Center, 651 Dongfeng Road East, Guangzhou 510060 , China. Email: zhangli63@hotmail.com; Hongyun Zhao. Department of Clinical Research, Sun Yat-sen University Cancer Center, 651 Dongfeng Road East, Guangzhou 510060, China. Email: zhaohy@sysucc.org.cn.

Background: The present study aimed to investigate the determinant factors of survival in patients with pretreated advanced stage non-small cell lung cancer (NSCLC) who received anti-PD-1/PD-L1 therapy.

Methods: In this observational retrospective study, the clinical profiles and laboratory parameters of patients with NSCLC treated with anti-PD-1/PD-L1 therapy were consecutively collected. Lung Immune Prognostic Index (LIPI) was calculated based on the derived neutrophil-to-lymphocyte ratio (dNLR) and lactate dehydrogenase level (LDH). Modified Glasgow Prognostic Score (mGPS) was calculated based on serum $\mathrm{C}$ reactive protein and albumin, and tumor mutation burden (TMB) was calculated using a targeted next-generation sequencing panel based on 422 cancer-relevant genes. The primary and secondary end points were overall survival (OS) and progression-free survival (PFS), respectively. The Cox regression model was used to identify the potential determinant factors of survival benefit. Trained oncologists at Sun Yat-sen University Cancer Center followed all of the participants through visits to doctors' offices or via telephone calls to determine their clinical status.

Results: Seventy-three patients were included in our study. With a median follow up time of 637 days, there was a significant difference in PFS between patients with high TMB compared to those with low TMB (3.7 vs. 2.1 months; $\mathrm{P}=0.004)$, while no significant difference was found in OS (14.0 vs. 16.4 months; $\mathrm{P}=0.972)$. Patients with a good LIPI score had a significantly longer OS compared to patients with a poor LIPI score (19.2 vs. 12.6 months; $\mathrm{P}=0.010$ ). The median $\mathrm{OS}$ in patients with a good and a poor mGPS was 16.8 and 4.3 months, respectively $(\mathrm{P}=0.029)$. In multivariate analysis, TMB was found to be significantly associated with PFS (HR, 0.38; 95\% CI: 0.21-0.69; P=0.002), while LIPI score was found to be significantly associated with OS (HR, 0.50; 95\% CI: 0.28-0.89; $\mathrm{P}=0.012$ ).

Conclusions: In the present study, LIPI score was a significant determinant of OS in patients with advanced NSCLC who received ICIs; however, TMB was only associated with PFS and not associated with OS.

Keywords: Non-small cell lung cancer (NSCLC); immune checkpoint inhibitors (ICIs); survival benefits; determinant factors; LIPI score; tumor mutation burden (TMB)

Submitted Apr 07, 2021. Accepted for publication Sep 30, 2021.

doi: $10.21037 / \mathrm{atm}-21-1702$

View this article at: https://dx.doi.org/10.21037/atm-21-1702 


\section{Introduction}

Recently, immunotherapy with immune checkpoint inhibitors (ICIs) has achieved excellent outcomes in the treatment of several types of cancer and was approved as the frontline treatment for patients with non-small cell lung cancer (NSCLC) $(1,2)$. The response rate for this treatment is still unsatisfactory. In pretreated patients with NSCLC, the response rate to anti-PD-1/PD-L1 monotherapy was seen in only $14-20 \%$ of patients. When these patients were stratified by PD-L1 expression level on tumor cells, the response improved to up to $30 \%$ in patients with strong PD-L1 expression (1). Tumor mutation burden (TMB) is considered to be a potential factor that is associated with ICIs treatment outcomes $(3,4)$. The predictive value of TMB is supported by the results from the KEYNOTE-158 trial, followed by its approval as a predictive biomarker for immunotherapy response in patients with different tumor types who were treated with pembrolizumab (5). Although PD-L1 expression and TMB have been incorporated into clinical practice as the most promising biomarkers for anti-PD-1/PDL1 treatment outcomes, not all patients with positive PD-L1 or high TMB can attain clinical benefits from immunotherapy; therefore, their predictive values require further optimization (6).

Peripheral blood-based biomarkers, such as inflammatory and nutritional-related markers, have emerged as potential biomarkers for immunotherapy outcomes $(7,8)$. In NSCLC, some novel prognostic scores, such as LIPI score and mGPS, have been evaluated for their association with anti-PD-1/PD-L1 therapy outcomes (9-12). These peripheral blood-based biomarkers have not been validated in large-scale prospective studies. In addition, the association between these biomarkers and TMB or PD-L1 is still not fully understood. Recent studies have demonstrated that multiple clinical factors, such as gender and smoking history, may influence the treatment outcomes of ICIs in patients with NSCLC $(13,14)$. The current study sought to identify the determinant factor of survival benefits in advanced NSCLC patients pretreated with ICIs. We incorporated multiple factors in a multivariable analyzing model, including patient characteristics, LIPI score, mGPS, and TMB. We present the following article in accordance with the recommendations for tumor marker prognostic studies (REMARK) reporting checklist (available at https://dx.doi. org/10.21037/atm-21-1702).

\section{Methods}

\section{Patients}

This observational retrospective study involved 73 patients with advanced NSCLC who received either anti-PD-1 (pembrolizumab, nivolumab, or camrelizumab) or antiPD-L1 (atezolizumab) therapy in a phase III control clinical trial at Sun Yat-sen University Cancer Center between December 2015 and August 2017. This study had a median follow up time of 637 days. All of the patients fulfilled the predefined inclusion criteria, including (I) age $>18$ years old; (II) performance status (ECOG) of $0-1$; (III) confirmed diagnosis of recurrent or metastatic NSCLC; and (IV) assessable tumor lesion by computed tomography or magnetic resonance imaging according to Response Evaluation Criteria in Solid Tumor (RECIST. v1.1). Tumor FFPE tissue sections $(n=58)$ or biopsy samples $(n=15)$ were obtained before treatment and used for genetic testing. Tumor driver mutation was detected using next-generation sequencing (NGS).

TMB was calculated using a targeted next-generation sequencing panel that was based on 422 cancer-relevant genes. This was done by adding alterations in the coding region of targeted genes, including base substitutions, indels, and synonymous alterations. As previously described, known driver mutations were excluded (15) with an average coverage depth and size of $1341 \mathrm{X}$ and $1.4 \mathrm{Mb}$, respectively. A cut-off of 10 mutations per megabase was used to classify patients into groups defined as high and low TMB. Peripheral blood samples were obtained from all of the patients before treatment. The derived neutrophil-to-lymphocyte ratio (dNLR) was calculated as the absolute neutrophil count/ (white blood cell - absolute neutrophil). LIPI score was calculated based on $\mathrm{dNLR}>3$ and LDH level $>$ the upper limit of normal, as previously reported (11). Due to the limited number of patients with a LIPI score of 2, we stratified patients into two groups: good LIPI score [0], and poor LIPI score [1-2] (16). The mGPS scores were calculated based on serum $\mathrm{C}$ reactive protein and albumin with cutoffs of $10 \mathrm{mg} / \mathrm{L}$ and $35 \mathrm{~g} / \mathrm{L}$, respectively. An mGPS score of $0-1$ and 2 were classified as good and poor mGPS scores, respectively (17). This study was conducted in accordance with the Declaration of Helsinki (as revised in 2013). This study was approved by the Institutional Review Board of Sun Yat-sen University Cancer Center (pembrolizumab: A2016019; camrelizumab: A2016-009; nivolumab: A2015-035; atezolizumab: A2016-021). In addition, individual consent for this retrospective analysis was waived. 
Table 1 Patients baseline characteristics

\begin{tabular}{|c|c|c|}
\hline Variables & Patients No. & $\%$ \\
\hline Gender (female/male) & $22 / 51$ & $30 / 70$ \\
\hline \multicolumn{3}{|l|}{ Age } \\
\hline Median & 54 & \\
\hline Range & $28-73$ & \\
\hline \multicolumn{3}{|l|}{ Pathological type } \\
\hline Squamous & 23 & 31.5 \\
\hline Non-squamous & 50 & 68.5 \\
\hline Smoking (yes/no) & $36 / 37$ & $49.3 / 50.7$ \\
\hline ECOG (0/1) & $22 / 51$ & $30.1 / 69.9$ \\
\hline \multicolumn{3}{|l|}{ Line of immunotherapy } \\
\hline $1 \mathrm{st}$ & 4 & 5.5 \\
\hline 2nd & 35 & 47.9 \\
\hline $3 r d$ & 18 & 24.7 \\
\hline 4th or above & 16 & 21.9 \\
\hline \multicolumn{3}{|c|}{ Pretreatment metastases } \\
\hline 1 & 19 & 26 \\
\hline 2 & 26 & 35.6 \\
\hline 3 & 18 & 24.7 \\
\hline 4 or more & 10 & 13.7 \\
\hline \multicolumn{3}{|l|}{ LIPI score } \\
\hline 0 & 36 & 49.3 \\
\hline 1 & 30 & 41.1 \\
\hline 2 & 7 & 9.6 \\
\hline \multicolumn{3}{|l|}{ mGPS } \\
\hline 0 & 1 & 1.4 \\
\hline 1 & 60 & 82.2 \\
\hline 2 & 12 & 16.4 \\
\hline \multicolumn{3}{|l|}{ TMB } \\
\hline High & 24 & 33 \\
\hline Low & 46 & 63 \\
\hline Unknown & 3 & 4 \\
\hline \multicolumn{3}{|l|}{ PD-L1 expression } \\
\hline Negative & 14 & 19.2 \\
\hline Positive & 13 & 17.8 \\
\hline Unknown & 46 & 63 \\
\hline
\end{tabular}

ECOG, eastern cooperative oncology group performance status; LIPI, lung immune prognostic index; mGPS, modified Glasgow Prognostic Score; TMB, tumor mutational burden.

\section{Treatments and response assessment}

All of the patients included in this study were treated as part of a phase III clinical trial. Most patients received antiPD1/PD-L1 as their second or later line of treatment after the standard frontline treatment had failed. PFS was defined as the time from treatment initiation to the date of disease progression. OS was defined as the time from the initiation of anti-PD1/PD-L1 treatment to the date of death from any cause. Treatment response evaluation was based on RECIST evaluation criteria (RECIST. v1.1). Trained oncologists at Sun Yat-sen University Cancer Center followed all of the participants through visits to doctors' offices or via telephone calls to determine their clinical status.

\section{Statistical analysis}

Predefined clinical endpoints in our analysis were PFS and OS. Normally distributed numeric variables are indicated by the mean $\pm \mathrm{SD}$ and categorical variables are indicated as a percentage (\%). Estimation of PFS and OS was determined by Kaplan-Meier analysis and the differences were examined by log-rank test. Cox regression analysis was used to determine the adjusted hazard ratio (HR) for PFS and OS. The gender, age, pathological type, smoking history, ECOG, baseline metastases number, prior treatment lines, LIPI, mGPS, and TMB for all of the patients were included in the univariate analysis. Variables with $\mathrm{P}$ value of $<0.10$ were further assessed in multivariable analysis. A two-sided $\mathrm{P}$ value of $<0.05$ was considered to be significant. All of the analyses were conducted with the use of SPSS version 20 (IBM SPSS Statistics, RRID:SCR_019096) and GraphPad software version 8 (GraphPad Prism, RRID:SCR_002798) was used to draw the figures.

\section{Results}

\section{Baseline characteristics of patients}

The baseline characteristics of patients are shown in Table 1. Overall, 95 patients received anti-PD-1/PD-L1 monotherapy. After excluding patients with ineligible WES results, 73 patients were included in the final analysis with a median age of 54 years. Fifty-one patients $(70 \%)$ were male and $36(49.3 \%)$ patients were current or former smokers. Fifty patients $(68.5 \%)$ were diagnosed with non-squamous NSCLC. TMB was high in $24(33 \%)$ patients. A total of 


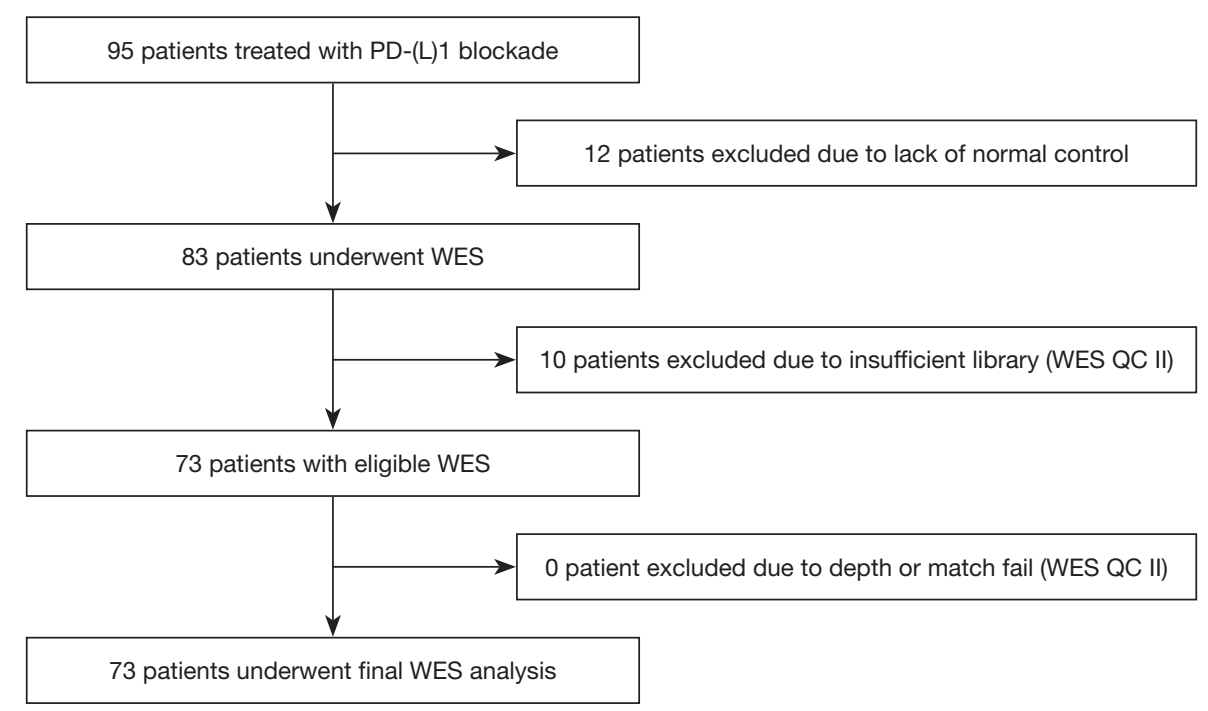

Figure 1 Flow chart illustrates the selection of eligible patients for the final analysis. WES, whole exome sequencing; QC, quality control.

36 (47\%) patients received anti-PD-1/PD-L1 after failure to second line treatment, and 54 (74\%) patients had 2 or more metastatic sites before treatment. Among all of the patients, $36(49.3 \%)$ and 61 (84\%) were classified as having a good score of LIPI and mGPS, respectively. The selection of eligible patients for the final analysis is illustrated with a flow chart in Figure 1.

\section{PFS and OS benefits analysis}

Overall, the median PFS was 2.1 months (95\% CI: 1.99-2.21) and median OS was 15.8 months (95\% CI: 10.90-20.30). Our results showed that the variables of male sex, smoking history, and high TMB were associated with a significantly longer PFS. The median PFS (mPFS) in males compared with females was 3.6 and 1.7 months, respectively (log-rank $\mathrm{P}=0.004)$. In patients who were current or former smokers and in non-smokers, mPFS was 4.2 and 1.9 months ( $\log$-rank $\mathrm{P}=0.003)$, respectively. Patients with high TMB and low TMB had an mPFS of 3.7 and 2.1 months (log-rank $\mathrm{P}=0.004)$, respectively. These results are shown in Figure $2 A$. In patients with both good and poor LIPI scores, mPFS was 3.7 and 2.0 months (logrank $\mathrm{P}=0.164)$, respectively. These results are shown in Figure $2 B$. OS analysis found that patients with good LIPI scores had a significantly longer OS as compared with those patients with poor LIPI scores (19.2 vs. 12.6 months, logrank $\mathrm{P}=0.010)$. These results are shown in Figure $3 \mathrm{~A}$. In patients with a good mGPS, OS was significantly increased as compared to those patients with a poor mGPS (16.8 vs. 4.3 months, log-rank $\mathrm{P}=0.029)$. However, the variables of patient gender, smoking history, and TMB were not associated with OS benefit. This is shown in Figure $3 B$ (all $\mathrm{P}>0.05)$.

\section{Determinants of survival benefits}

Our subsequent analysis was focused on determining factors that could predict survival benefits. In univariate analysis of PFS, TMB was found to be a significant determinant of PFS (HR, 0.46; 95\% CI, 0.23-0.92; $\mathrm{P}=0.027)$. LIPI score was the second determinant of PFS benefit with a margin $\mathrm{P}$ value of 0.064 (HR, 0.59; $95 \%$ CI, 0.34-1.03). In multivariable analysis, TMB was identified as an independent determinant factor of PFS (HR, 0.41; 95\% CI, 0.23-0.73; $\mathrm{P}=0.002$ ), whereas LIPI was another independent determinant factor with a marginal $\mathrm{P}=0.081$ (HR, 0.64; 95\% CI, 0.38-1.06) (Table 2). When considering OS benefit, only LIPI was identified as a determinant factor of OS and remained significant in multivariable analysis (HR, 0.49; 95\% CI, 0.28-0.85; $\mathrm{P}=0.012$ ) (Table 3).

\section{Discussion}

In the current study, we aimed to investigate the potential determinant factors of ICIs as well as the different treatment outcomes in pretreated advanced or metastatic NSCLC patients who received PD-1/PD-L1 blockade therapy. 

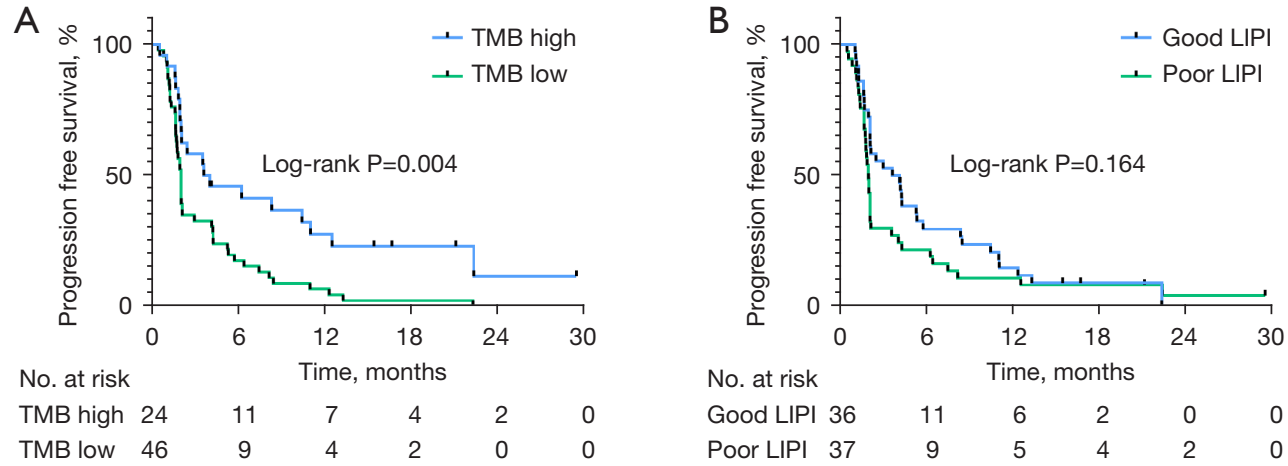

Figure 2 Kaplan-Meier estimates of PFS according to TMB (A) and LIPI score (B). PFS, progression-free survival; TMB, tumor mutational burden; LIPI, Lung Immune Prognostic Index.
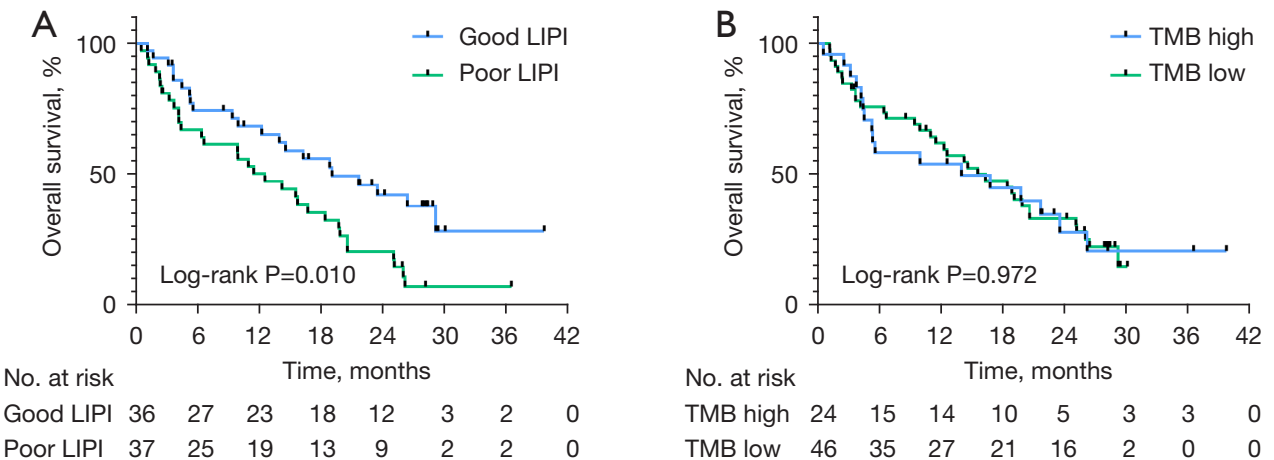

Figure 3 Kaplan-Meier estimates of OS according to LIPI score (A) and TMB (B). OS, overall survival; LIPI, Lung Immune Prognostic Index; TMB, tumor mutational burden.

Our analysis showed that male sex, smoking history, and TMB were associated with a significantly longer PFS; however, these factors were not associated with OS benefit. In addition, patients with a good LIPI score had a longer PFS as compared to patients who had a poor LIPI score with a marginal statistical difference. Interestingly, patients with a good LIPI score had a significantly longer OS than patients with a poor LIPI score. Similarly, patients who had a good mGPS before treatment had a longer OS compared to patients with poor mGPS. Furthermore, our analysis has confirmed the role of TMB as a significant determinant of ICIs for PFS and LIPI score as an independent determinant factor of OS benefit.

The introduction of immunotherapy with anti-PD-1/ PD-L1 led to a major advancement in the treatment of NSCLC. Treatment with either ICIs in monotherapy or in combination with chemotherapy has been approved as the first-line treatment option in patients with NSCLC without actionable tumor driver mutations (18). However, the response rate of ICIs therapies is still lower than anticipated. Several factors have been extensively evaluated to determine their roles as treatment biomarkers. These include TMB and PD-L1 expression in tumor bed (19). Consistent with previous studies, our results showed that high TMB was significantly associated with improved PFS $(20,21)$; however, the role of TMB as a predictive marker of OS in patients treated with ICIs remains unclear. In our study, TMB was not found to be associated with OS benefit. Similar results were reported in a recent metaanalysis, which suggests that TMB was not associated with OS benefit (22). Thus, the predictive value of TMB in determining OS benefit warrants further investigation.

Although TMB has a promising predictive value for ICIs treatment outcomes, its utility in clinical practice is constrained due to several limitations, including the lack of an optimal cutoff value across different tumor types. 
Table 2 Univariable and multivariable analysis of progression free survival

\begin{tabular}{|c|c|c|c|c|}
\hline Variable & \multicolumn{2}{|c|}{ Univariate } & \multicolumn{2}{|c|}{ Multivariate } \\
\hline Gender (male vs. female) & $0.74(0.36-1.52)$ & 0.412 & - & - \\
\hline Age ( $\geq 60$ vs. $<60$ years) & $1.36(0.76-2.45)$ & 0.303 & - & - \\
\hline Pathological type (SCC vs. non-SCC) & $0.98(0.54-1.80)$ & 0.954 & - & - \\
\hline ECOG (0 vs. 1) & $1.26(0.68-2.35)$ & 0.463 & - & - \\
\hline Line of immunotherapy ( $\leq 2 \mathrm{nd}$ vs. $>2 \mathrm{nd}$ ) & $1.14(0.65-2.03)$ & 0.642 & - & - \\
\hline Pretreatment metastases ( $\geq 2$ vs. $<2$ ) & $0.91(0.47-1.76)$ & 0.787 & - & - \\
\hline LIPI score (good vs. poor) & $0.59(0.34-1.03)$ & 0.064 & $0.64(0.38-1.06)$ & 0.081 \\
\hline
\end{tabular}

SCC, squamous cell carcinoma; non-SCC, non-squamous cell carcinoma; ECOG, eastern cooperative oncology group performance status; LIPI, lung immune prognostic index; mGPS, modified Glasgow Prognostic Score; TMB, tumor mutational burden; HR, hazard ratio; $\mathrm{Cl}$, confidence interval.

Table 3 Univariable and multivariable analysis of overall survival

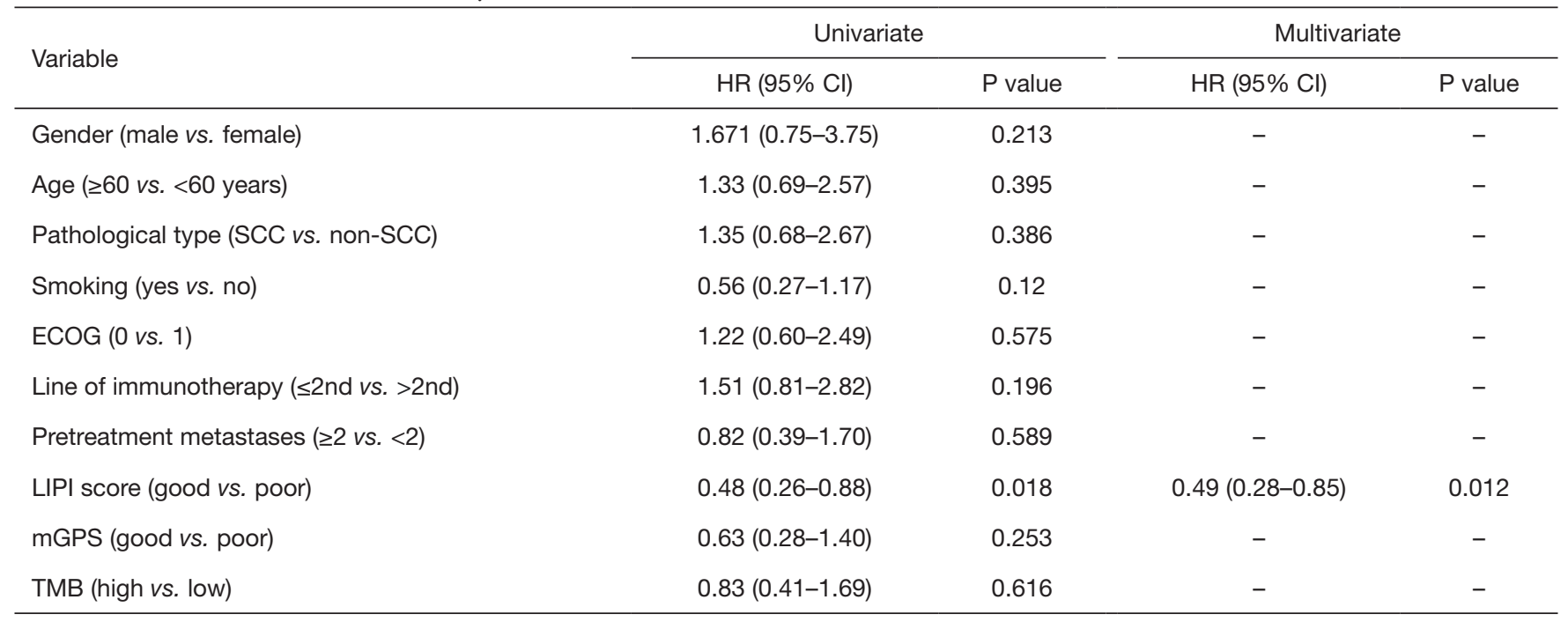

SCC, squamous cell carcinoma; non-SCC, non-squamous cell carcinoma; ECOG, eastern cooperative oncology group performance status; LIPI, lung immune prognostic index; mGPS, modified Glasgow Prognostic Score; TMB, tumor mutational burden; HR, hazard ratio; $\mathrm{Cl}$, confidence interval.

In addition, its significance as a single biomarker for ICI treatment outcomes might be sub-optimal $(6,23)$. Recently, the association of LIPI score with ICI treatment outcomes has been investigated in the setting of multiple cancers, including NSCLC (24). In NSCLC patients who received ICIs as the first-line therapy, LIPI score was identified as an independent prognostic factor $(25,26)$. In the present study, we found that LIPI score was a significant determinant of OS irrespective of TMB; however, due to the limited number of patients with a LIPI score of 2, we classified patients into two groups: good LIPI score [0], and poor LIPI score [1-2]. 
Of note, the mechanism for anti-tumor activity of ICIs may be due to these inhibitors breaking the inhibitory signals of T-cell activation against tumors (27). TMB can serve as a surrogate of anti-tumor immunity that is elicited by tumor neoantigens, whereas immune reaction may be reflected by a specific immune cell that is in abundance in the tumor microenvironment or peripheral blood. Our analysis results showed that the prognostic value of LIPI was independent of TMB. A combination of TMB with LIPI score might be used as surrogate biomarkers to more significantly identify patients with survival benefits as compared with either TMB or LIPI score as a single predictor $(28,29)$. Interestingly, when we compared patients with high TMB and good LIPI score versus patients with low TMB and poor LIPI score, a more statistically significant difference was seen in mPFS compared with patients stratified by TMB alone. However, there were only a limited number of patients in each group. These results indicate that the combination of TMB and LIPI score as a potential biomarker for ICI response is worth further investigation.

Our study has some limitations, since it is retrospective in nature and was conducted with a relatively small patient population. However, the patients included in our analysis were enrolled in a controlled clinical trial, which reduced the bias of patient selection. PD-L1 expression was available only for a small number of patients, so this was not included in the multivariable analysis of treatment determinant factors. A recent study has shown that the prognostic value of LIPI score is independent of PD-L1 expression (30). Another limitation is that our analysis included some patients with EGFR mutation ( $\mathrm{n}=5$ ) or ALK rearrangement $(\mathrm{n}=1)$. Patients with these features have been shown to respond poorly to ICIs.

To the best of our knowledge, this is the first study to include mGPS, LIPI score, and TMB in a multivariable analysis of survival benefit in patients with NSCLC who have previously received ICI therapy. Although the prognostic value of LIPI score and mGPS have been reported previously, earlier studies did not include TMB in their multivariable analysis model of outcomes. This may affect the prognostic role of LIPI score or mGPS. Our study showed that the prognostic value of LIPI score was independent of TMB. Although TMB is a promising biomarker for immunotherapy outcomes, it still presents several limitations. In the present study, LIPI score was a promising biomarker of overall survival (OS) in patients with advanced NSCLC who received ICIs. TMB was associated with progression-free survival (PFS), but not OS. LIPI score represented a readily available biomarker for immunotherapy outcomes; however, the role of LIPI score as a prognostic biomarker for ICIs treatment outcomes requires further validation in future studies.

\section{Acknowledgments}

We would like to thank LetPub (https://www.letpub.com/) and Abdul-Quddus Mohammed for their help in polishing our paper.

Funding: This research was supported by Guangdong Basic and Applied Basic Research Foundation [2020A1515010020 for YM and 2018A0303130243 for HZ] and National Nature Science Foundation of China [82002409 for YM, 81872201 for HZ and 81872499 for LZ].

\section{Footnote}

Reporting Checklist: The authors have completed the REMARK reporting checklist. Available at https://dx.doi. org/10.21037/atm-21-1702

Data Sharing Statement: Available at https://dx.doi. org/10.21037/atm-21-1702

Conflicts of Interest: All authors have completed the ICMJE uniform disclosure form (available at https://dx.doi. org/10.21037/atm-21-1702). The authors have no conflicts of interest to declare.

Ethical Statement: The authors are accountable for all aspects of the work in ensuring that questions related to the accuracy or integrity of any part of the work are appropriately investigated and resolved. This study was conducted in accordance with the Declaration of Helsinki (as revised in 2013). This study was approved by the Institutional Review Board of Sun Yat-sen University Cancer Center (pembrolizumab: A2016-019; camrelizumab: A2016-009; nivolumab: A2015-035; atezolizumab: A2016021), and individual consent for this retrospective analysis was waived.

Open Access Statement: This is an Open Access article distributed in accordance with the Creative Commons Attribution-NonCommercial-NoDerivs 4.0 International License (CC BY-NC-ND 4.0), which permits the noncommercial replication and distribution of the article with 
the strict proviso that no changes or edits are made and the original work is properly cited (including links to both the formal publication through the relevant DOI and the license). See: https://creativecommons.org/licenses/by-nc-nd/4.0/.

\section{References}

1. Doroshow DB, Sanmamed MF, Hastings K, et al. Immunotherapy in Non-Small Cell Lung Cancer: Facts and Hopes. Clin Cancer Res 2019;25:4592-602.

2. Waldman AD, Fritz JM, Lenardo MJ. A guide to cancer immunotherapy: from $T$ cell basic science to clinical practice. Nat Rev Immunol 2020;20:651-68.

3. Chan TA, Yarchoan M, Jaffee E, et al. Development of tumor mutation burden as an immunotherapy biomarker: utility for the oncology clinic. Ann Oncol 2019;30:44-56.

4. Gjoerup O, Brown CA, Ross JS, et al. Identification and Utilization of Biomarkers to Predict Response to Immune Checkpoint Inhibitors. AAPS J 2020;22:132.

5. Marabelle A, Fakih M, Lopez J, et al. Association of tumour mutational burden with outcomes in patients with advanced solid tumours treated with pembrolizumab: prospective biomarker analysis of the multicohort, openlabel, phase 2 KEYNOTE-158 study. Lancet Oncol 2020;21:1353-65.

6. Wood MA, Weeder BR, David JK, et al. Burden of tumor mutations, neoepitopes, and other variants are weak predictors of cancer immunotherapy response and overall survival. Genome Med 2020;12:33.

7. Buder-Bakhaya K, Hassel JC. Biomarkers for Clinical Benefit of Immune Checkpoint Inhibitor Treatment-A Review From the Melanoma Perspective and Beyond. Front Immunol 2018;9:1474.

8. Voong KR, Feliciano J, Becker D, et al. Beyond PD-L1 testing-emerging biomarkers for immunotherapy in nonsmall cell lung cancer. Ann Transl Med 2017;5:376.

9. Kazandjian D, Gong Y, Keegan P, et al. Prognostic Value of the Lung Immune Prognostic Index for Patients Treated for Metastatic Non-Small Cell Lung Cancer. JAMA Oncol 2019;5:1481-5.

10. Maymani H, Hess K, Groisberg R, et al. Predicting outcomes in patients with advanced non-small cell lung cancer enrolled in early phase immunotherapy trials. Lung Cancer 2018;120:137-41.

11. Mezquita L, Auclin E, Ferrara R, et al. Association of the Lung Immune Prognostic Index With Immune Checkpoint Inhibitor Outcomes in Patients With Advanced Non-Small Cell Lung Cancer. JAMA Oncol
2018;4:351-7.

12. Takamori S, Takada K, Shimokawa M, et al. Clinical utility of pretreatment Glasgow prognostic score in non-smallcell lung cancer patients treated with immune checkpoint inhibitors. Lung Cancer 2021;152:27-33.

13. Lichtenstein MRL, Nipp RD, Muzikansky A, et al. Impact of Age on Outcomes with Immunotherapy in Patients with Non-Small Cell Lung Cancer. J Thorac Oncol 2019;14:547-52.

14. Pinto JA, Vallejos CS, Raez LE, et al. Gender and outcomes in non-small cell lung cancer: an old prognostic variable comes back for targeted therapy and immunotherapy? ESMO Open 2018;3:e000344.

15. Fang W, Ma Y, Yin JC, et al. Comprehensive Genomic Profiling Identifies Novel Genetic Predictors of Response to Anti-PD-(L)1 Therapies in Non-Small Cell Lung Cancer. Clin Cancer Res 2019;25:5015-26.

16. Zhang T, Xue W, Wang D, et al. A validation study on the lung immune prognostic index for prognostic value in patients with locally advanced non-small cell lung cancer. Radiother Oncol 2021;156:244-50.

17. McMillan DC. The systemic inflammation-based Glasgow Prognostic Score: a decade of experience in patients with cancer. Cancer Treat Rev 2013;39:534-40.

18. Lim SM, Hong MH, Kim HR. Immunotherapy for Nonsmall Cell Lung Cancer: Current Landscape and Future Perspectives. Immune Netw 2020;20:e10.

19. Havel JJ, Chowell D, Chan TA. The evolving landscape of biomarkers for checkpoint inhibitor immunotherapy. Nat Rev Cancer 2019;19:133-50.

20. Forde PM, Chaft JE, Smith KN, et al. Neoadjuvant PD-1 Blockade in Resectable Lung Cancer. N Engl J Med 2018;378:1976-86.

21. Rizvi NA, Hellmann MD, Snyder A, et al. Cancer immunology. Mutational landscape determines sensitivity to PD-1 blockade in non-small cell lung cancer. Science 2015;348:124-8.

22. Osipov A, Lim SJ, Popovic A, et al. Tumor Mutational Burden, Toxicity, and Response of Immune Checkpoint Inhibitors Targeting PD(L)1, CTLA-4, and Combination: A Meta-regression Analysis. Clin Cancer Res 2020;26:4842-51.

23. Strickler JH, Hanks BA, Khasraw M. Tumor Mutational Burden as a Predictor of Immunotherapy Response: Is More Always Better? Clin Cancer Res 2021;27:1236-41.

24. Benitez JC, Recondo G, Rassy E, et al. The LIPI score and inflammatory biomarkers for selection of patients with solid tumors treated with checkpoint inhibitors. Q J Nucl 
Med Mol Imaging 2020;64:162-74.

25. Blanc-Durand F, Auclin E, Planchard D, et al. Association of lung immune prognostic index (LIPI) with survival of first line immune checkpoint inhibitors single agent or in combination with chemotherapy in untreated advanced NSCLC patients. Ann Oncol 2019;30:xi5.

26. Mielgo Rubio X, Gomez Rueda A, Antoñanzas M, et al. Applicability of lung immune prognostic index (LIPI) to predict efficacy of first-line pembrolizumab in advanced non-small cell lung cancer (NSCLC). Ann Oncol 2019;30:v619-v620.

27. Wei SC, Duffy CR, Allison JP. Fundamental Mechanisms of Immune Checkpoint Blockade Therapy. Cancer Discov
2018;8:1069-86.

28. Huang AC, Postow MA, Orlowski RJ, et al. T-cell invigoration to tumour burden ratio associated with antiPD-1 response. Nature 2017;545:60-5.

29. Lesterhuis WJ, Bosco A, Millward MJ, et al. Dynamic versus static biomarkers in cancer immune checkpoint blockade: unravelling complexity. Nat Rev Drug Discov 2017;16:264-72.

30. Mezquita L, Park W, Arbour K, et al. P1.01-68 Correlation of the Lung Immune Prognostic Index (LIPI) and PDL1 Status with Outcomes for Immune Checkpoint Inhibitors in Advanced NSCLC Patients. J Thorac Oncol 2018; $13:$ S488.

Cite this article as: Ali WAS, Hui P, Ma Y, Wu Y, Zhang Y, Chen Y, Hong S, Yang Y, Huang Y, Zhao Y, Fang W, Zhao H, Zhang L. Determinants of survival in advanced non-small cell lung cancer patients treated with anti-PD-1/PD-L1 therapy. Ann Transl Med 2021;9(22):1639. doi: 10.21037/atm-21-1702 